\title{
DECAY ESTIMATES FOR SOLUTIONS OF NONLOCAL SEMILINEAR EQUATIONS
}

\author{
MARCO CAPPIELLO, \\ TODOR GRAMCHEV, AND LUIGI RODINO
}

\begin{abstract}
We investigate the decay for $|x| \rightarrow \infty$ of weak Sobolev-type solutions of semilinear nonlocal equations $P u=F(u)$. We consider the case when $P=$ $p(D)$ is an elliptic Fourier multiplier with polyhomogeneous symbol $p(\xi)$, and we derive algebraic decay estimates in terms of weighted Sobolev norms. Our basic example is the celebrated Benjamin-Ono equation
\end{abstract}

$$
(|D|+c) u=u^{2}, \quad c>0,
$$

for internal solitary waves of deep stratified fluids. Their profile presents algebraic decay, in strong contrast with the exponential decay for KdV shallow water waves.

\section{$\S 1$. Introduction}

The main goal of the present article is to investigate the appearance of algebraic decay at infinity for weak solutions of semilinear nonlocal elliptic equations of the form

$$
P u=F(u),
$$

where $P=p(D)$ is a Fourier multiplier in $\mathbb{R}^{n}$

$$
P u(x)=\int_{\mathbb{R}^{n}} e^{i x \xi} p(\xi) \hat{u}(\xi) d \xi,
$$

with $\hat{u}(\xi)=\int_{\mathbb{R}^{n}} e^{-i x \xi} u(x) d x, \overrightarrow{d \xi}=(2 \pi)^{-n} d \xi$, and where $F(u)$ is a polynomial vanishing of order $k \geq 2$ at $u=0$, namely,

$$
F(u)=\sum_{j=2}^{N} F_{j} u^{j}, \quad F_{j} \in \mathbb{C} .
$$

Received June 20, 2013. Revised July 25, 2014. Accepted July 27, 2014.

2010 Mathematics Subject Classification. Primary 35J61; Secondary 35B40, 35S05, $35 \mathrm{Q} 51$.

(C) 2015 by The Editorial Board of the Nagoya Mathematical Journal 
For $P$ an operator with constant coefficients, that is, a $p(\xi)$ polynomial, or the more general Fourier multiplier, equations of the form (1.1) arise frequently in mathematical physics in the theory of solitary waves for nonlinear evolution equations. Relevant examples are equations in the realm of wave motions featuring both dispersion and diffusion processes, long internal waves and the interface between two fluids of different densities, and semilinear Schrödinger equations. Let us recall, in short, the case $x \in \mathbb{R}$ : starting from an evolution equation of the form $v_{t}+(P v)_{x}=F(v)_{x}$, with $t \geq 0$, solitary waves are solutions of the form $v(t, x)=u(x-c t), c>0$. Looking for these types of solutions, one is indeed reduced to studying the elliptic equation (1.1).

There are no general methods for deriving the existence of such special solutions, and in the known examples special features like conservation laws and/or the presence of symmetries play a fundamental role. On the other hand, it is natural to study regularity and behavior at infinity of these types of waves in order to have a global knowledge of their profile. In the fundamental papers [5] and [6], Bona and Li proved that if $p(\xi)$ is analytic on $\mathbb{R}$, then every solution $u \in L^{\infty}(\mathbb{R})$ of (1.1) such that $u(x) \rightarrow 0$ for $x \rightarrow \pm \infty$ exhibits an exponential decay of the form $e^{-\varepsilon|x|}, \varepsilon>0$ for $|x| \rightarrow \infty$, and extends to a holomorphic function in a strip of the form $\{z \in \mathbb{C}:|\Im z|<T\}$ for some $T>0$. The research efforts in [5] and [6] were motivated by the applications to the study of decay and analyticity of solitary waves for $\mathrm{KdV}$ type, long-wave-type, and Schrödinger-type equations. In [4] and [9], we proved similar results in arbitrary dimension for analytic pseudodifferential operators, deriving sharp estimates in the frame of the Gelfand-Shilov spaces of type $\mathcal{S}$ (see [19]), which give simultaneous information on the exponential decay at infinity and the Gevrey-analytic regularity on $\mathbb{R}^{n}$. Recently, the results on the holomorphic extensions have been refined in [11] and [12].

Here we want to consider the case when $p(\xi)$ is only finitely smooth at $\xi=0$. Namely, the symbol $p(\xi)$ is assumed to be a sum of positively homogeneous terms, and we are interested in the nonlocal case; that is, at least one of these terms is not a polynomial, hence $p(\xi)$ is only finitely smooth at the origin. In this case, the functional analytic machinery and the pseudodifferential calculus used in the above-mentioned papers are not applicable. Motivation for this type of study comes from two directions. The first is the presence of several nonlinear models in the theory of solitary waves in which the symbol of the linear part presents singularities or finite smoothness at $\xi=0$. The most celebrated equation in this category is the 
so-called Benjamin-Ono equation (0.1) (see [2], [3], [22], [25], [28]), which will be considered in detail in Section 2.

Another more general issue comes from the novelty with respect to the general theory on decay and regularity estimates for linear and nonlinear elliptic equations in $\mathbb{R}^{n}$ (besides [4]-[6], [9], [11], and [12], see, e.g., [1], [10], [13], [26], [24], and [29]). In fact, we are not aware of any result for general semilinear elliptic equations of the form (1.1) in the case of finitely smooth symbols. As a first step, in this paper we will focus on the decay of the solutions with the purpose of treating analytic regularity and holomorphic extensions in a future work. With respect to the case of smooth or analytic symbols, a finite smoothness of the symbol of the linear part of (1.1) may determine the loss of the rapid or exponential decay observed in all the above-mentioned publications. This fact is confirmed by several examples (see Section 2 below). As a novelty, we can prove that in this new situation the solutions of (1.1) present at least an algebraic decay at infinity whose rate depends on the dimension $n$ and on the regularity of the symbol $p(\xi)$. This decay will be proved in terms of estimates in the weighted Sobolev spaces

$$
H^{s, t}\left(\mathbb{R}^{n}\right):=\left\{u \in \mathcal{S}^{\prime}\left(\mathbb{R}^{n}\right):\|u\|_{s, t}=\left\|\langle x\rangle^{t}\langle D\rangle^{s} u\right\|_{L^{2}\left(\mathbb{R}^{n}\right)}<\infty\right\}, \quad s, t \in \mathbb{R},
$$

where $\langle x\rangle=\left(1+|x|^{2}\right)^{1 / 2}$ and $\langle D\rangle^{s}$ denotes the multiplier with symbol $\langle\xi\rangle^{s}$. Note that, for $t=0, H^{s, 0}\left(\mathbb{R}^{n}\right)$ coincides with the standard Sobolev space $H^{s}\left(\mathbb{R}^{n}\right)$. We will denote as standard by $\|\cdot\|_{s}$ the norm $\|\cdot\|_{s, 0}$. (We refer the reader to [13] for a detailed presentation of the properties of these spaces.)

Let us now detail the class of operators $p(D)$ to which our results apply. We will consider Fourier multipliers with symbols of the type

$$
p(\xi)=p_{0}+\sum_{j=1}^{h} p_{m_{j}}(\xi)
$$

where $p_{0} \in \mathbb{C}$ and $p_{m_{j}}(\xi) \in C^{\infty}\left(\mathbb{R}^{n} \backslash 0\right)$ are (positively) homogeneous symbols of order $m_{j}$, that is, $p_{m_{j}}(\lambda \xi)=\lambda^{m_{j}} p(\xi)$ for $\lambda>0$, with $0<m_{1}<m_{2}<$ $\cdots<m_{h}=M$. We assume that $M \geq 1$ and that the following global ellipticity condition holds:

$$
\inf _{\xi \in \mathbb{R}^{n}}\left(\langle\xi\rangle^{-M}|p(\xi)|\right)>0
$$

Since $p(0)=p_{0}$, condition (1.5) implies in particular that $p_{0} \neq 0$. 
Moreover, set

$$
m:=\min \left\{m_{j}: p_{m_{j}} \text { is not polynomial }\right\} .
$$

We will call $m$ the singularity index of $p(\xi)$. When the set in the righthand side of (1.6) is empty, then $P=p(D)$ is a partial differential operator with constant coefficients, and we go back to the above-mentioned results of exponential decay.

Our main result is the following.

TheOREM 1.1. Let $m \in \mathbb{R}$ with $[m]>n / 2$, and let $P$ be an operator with symbol $p(\xi)$ of the form (1.4) and (1.6) satisfying the assumption (1.5). Assume that $u$ is a solution of (1.1) such that $u \in H^{s, \varepsilon_{o}}\left(\mathbb{R}^{n}\right)$ for some $s>$ $n / 2$ and for some $\varepsilon_{o}>0$. Then, $u \in C^{\infty}\left(\mathbb{R}^{n}\right)$, and for every $\alpha \in \mathbb{N}^{n}$ and $\varepsilon>0$ we have

$$
\partial^{\alpha} u \in H^{s,|\alpha|+m+n / 2-\varepsilon}\left(\mathbb{R}^{n}\right) ;
$$

that is, the following estimate holds:

$$
\left\|\langle x\rangle^{m+n / 2-\varepsilon} x^{\beta} \partial^{\alpha} u\right\|_{s}<\infty
$$

for every $\alpha, \beta \in \mathbb{N}^{n}$, with $|\beta| \leq|\alpha|$. Under the same assumptions on $p(D)$ and $u$, the same result holds for solutions of the equation

$$
p(D) u=f+F(u),
$$

where $f$ is a given smooth function satisfying (1.7).

We observe that the second part of Theorem 1.1 turns out to be new also for linear equations, that is, when $F(u)=0$ in (1.8), whereas the first part is trivial in this case since the homogeneous equation $p(D) u=0$ admits only the solution $u=0$, the nonlinearity being essential to produce nontrivial solutions when $f=0$. Note also that the estimate (1.7) implies that

$$
\left\|\langle x\rangle^{m+n / 2-\varepsilon} u\right\|_{L^{2}}<\infty .
$$

Unfortunately, by our methods we are not able to prove the stronger result

$$
\left\||\cdot|^{m+n} u\right\|_{L^{\infty}\left(\mathbb{R}^{n}\right)}<\infty .
$$

Such pointwise estimates have been obtained in [25] for a class of BenjaminOno-type equations and are satisfied by the solutions of other similar models described in Section 2 (see also [15], [14], and [20] for further results on algebraic decay at infinity for dispersive equations). 
REMARK 1. In Theorem 1.1, we assume that $u \in H^{s, \varepsilon_{o}}\left(\mathbb{R}^{n}\right)$ for some $s>$ $n / 2$ and for some $\varepsilon_{o}>0$. This is a technical condition needed in our general setting for the proof of Lemma 4.1. In some special cases the assumption can be weakened (see, e.g., [25, Theorem 4] for the generalized Benjamin-Ono equation).

Also, the condition $[m]>n / 2$ is technical. It is used in the inductive scheme in Section 4, but it does not seem necessary for the validity of the estimate (1.9) (see Example 3 and Remark 3 in Section 2).

\section{§2. Examples}

This section is devoted to the analysis of some examples of nonlocal semilinear equations whose solutions admit algebraic decay of the form (1.9). In particular, we will test our decay estimates on these models. The first and more important example is given by the Benjamin-Ono equation in hydrodynamics.

ExAmple 1. The Benjamin-Ono equation, introduced in [3] and [28], describes 1-dimensional internal waves in stratified fluids of great depth. It reads as follows:

$$
\partial_{t} v+H\left(\partial_{x}^{2} v\right)+2 v \partial_{x} v=0, \quad t \in \mathbb{R}, x \in \mathbb{R},
$$

where $H(D)$ stands for the Hilbert transform, that is, the Fourier multiplier operator of order 0 with symbol $-i \operatorname{sign} \xi$ :

$$
H(D) u(x)=\frac{1}{\pi} \mathrm{P} \cdot \mathrm{V} \cdot \int_{\mathbb{R}} \frac{u(y)}{x-y} d y=\int_{\mathbb{R}} e^{i x \xi}(-i \operatorname{sign} \xi) \hat{u}(\xi) d \xi .
$$

There exists a large number of articles dealing with existence, uniqueness, and time asymptotics for the initial value problem related to the equation (2.1) and its generalizations in various functional settings (see, e.g., [7], [8], [17], [23], [25], [27], [30], [32]). Concerning the solitary waves $u(x-c t), c>0$, they satisfy the nonlocal elliptic equation (0.1) which corresponds to (1.1) for $p(\xi)=|\xi|+c$ and $F(u)=u^{2}$. In [3], Benjamin found the solution

$$
u(x)=\frac{2 c}{1+c^{2} x^{2}}, \quad x \in \mathbb{R}
$$

(see (2.11) in Remark 2 for the easy computation). Later, Amick and Toland [2] proved that, apart from translations, the function (2.3) is the only solution of (0.1) which tends to 0 for $|x| \rightarrow \infty$. Note that $u(x)$ in (2.3) exhibits 
a quadratic decay at infinity like $|x|^{-2}$, satisfying (1.9) with $m=1, n=1$. (For other results and generalizations in higher dimension, see [25].)

The next example is not related to applicative problems and shows that polynomial terms $p_{m_{j}}(\xi)$ in the expression of $p(\xi)$ have no influence on the rate of decay.

EXAMPLE 2. In dimension $n=1$, consider the equation

$$
-u^{\prime \prime}+3|D| u+3 u=8 u^{3}
$$

where $|D|$ is as before the Fourier multiplier with symbol $|\xi|, \xi \in \mathbb{R}$. Note that the linear part of (2.4) is globally elliptic, that is, (1.5) is satisfied. Moreover, the order is $M=2$ and the singularity index is $m=1$. We have the following result.

Proposition 2.1. The equation (2.4) admits the solution

$$
u(x)=\frac{1}{1+x^{2}}, \quad x \in \mathbb{R} .
$$

Proof. We will check that the Fourier transforms of the left- and righthand sides of (2.4) coincide for $u(x)$ as in (2.5). To this end, we recall (see, e.g., [31, (VII, 7;23), p. 260] or [18, (9), p. 187]) that

$$
\mathcal{F}\left(\left(1+|x|^{2}\right)^{-\lambda}\right)(\xi)=\frac{2 \pi^{n / 2}}{\Gamma(\lambda)}\left(\frac{|\xi|}{2}\right)^{\lambda-n / 2} K_{\lambda-n / 2}(|\xi|),
$$

where $x, \xi \in \mathbb{R}^{n}$ and where $\Gamma$ denotes the standard Euler function; arguing in the distribution sense, we may allow any $\lambda>0$. The functions $K_{\nu}(x), \nu \in \mathbb{R}$, $x \in \mathbb{R} \backslash 0$, are the modified Bessel functions of second type (for definitions and properties, see, e.g., [16], [33]). We recall in particular that

$$
\begin{aligned}
K_{\nu}(x) & =K_{-\nu}(x), \quad \nu \in \mathbb{R}, x \neq 0, \\
K_{\nu+1}(x) & =\frac{2 \nu}{x} K_{\nu}(x)+K_{\nu-1}(x), \quad \nu \in \mathbb{R}, x \neq 0 .
\end{aligned}
$$

From (2.7), (2.8), we have

$$
\begin{aligned}
& K_{3 / 2}(x)=\left(\frac{1}{x}+1\right) K_{1 / 2}(x), \quad x \neq 0, \\
& K_{5 / 2}(x)=\left(\frac{3}{x^{2}}+\frac{3}{x}+1\right) K_{1 / 2}(x), \quad x \neq 0 .
\end{aligned}
$$


Let us then prove that (2.5) is a solution of (2.4). In fact, from (2.6) and (2.10), we have

$$
\begin{aligned}
8 \mathcal{F}\left(u^{3}\right) & =8 \mathcal{F}\left(\left(1+x^{2}\right)^{-3}\right) \\
& =8 \sqrt{\pi}\left(\frac{|\xi|}{2}\right)^{5 / 2} K_{5 / 2}(|\xi|) \\
& =2 \sqrt{\pi}\left(\xi^{2}+3|\xi|+3\right)\left(\frac{|\xi|}{2}\right)^{1 / 2} K_{1 / 2}(|\xi|) \\
& =\left(\xi^{2}+3|\xi|+3\right) \mathcal{F}\left(\left(1+x^{2}\right)^{-1}\right) \\
& =\mathcal{F}\left(D^{2} u+3|D| u+3 u\right) .
\end{aligned}
$$

REMARK 2. The method used in the proof of Proposition 2.1 can also be applied to the Benjamin-Ono equation and gives an easy alternative proof that the function $u(x)$ in (2.3) is a solution of (0.1), say, for $c=1$. In fact, from (2.6) and (2.9) we easily obtain

$$
\begin{aligned}
\mathcal{F}\left(u^{2}\right) & =4 \mathcal{F}\left(\left(1+x^{2}\right)^{-2}\right) \\
& =8 \sqrt{\pi}\left(\frac{|\xi|}{2}\right)^{3 / 2} K_{3 / 2}(|\xi|) \\
& =4 \sqrt{\pi}(|\xi|+1)\left(\frac{|\xi|}{2}\right)^{1 / 2} K_{1 / 2}(x) \\
& =2(|\xi|+1) \mathcal{F}\left(\left(1+x^{2}\right)^{-1}\right) \\
& =\mathcal{F}(|D| u+u) .
\end{aligned}
$$

Note that, by (2.8), we may calculate inductively $K_{N / 2}(x)$ for any odd integer $N$ in terms of $K_{1 / 2}(x)$. This allows us to produce other similar examples, with higher-order $M$, with $m=1$ and higher-order nonlinearity. Solutions are still of the form $u(x)=\frac{1}{1+x^{2}}$.

By the same argument, we obtain the following example in higher dimension.

EXAMPLE 3. In dimension $n=3$, consider the equation

$$
-\Delta u+3 \sqrt{-\Delta} u+3 u=24 u^{2}
$$


where $\sqrt{-\Delta}$ denotes the Fourier multiplier with symbol $|\xi|$. Note that the linear part of (2.12) is globally elliptic, that is, (1.5) is satisfied and the singularity index is $m=1$. The equation (2.12) admits the solution

$$
u(x)=\frac{1}{\left(1+|x|^{2}\right)^{2}}, \quad x \in \mathbb{R}^{3},
$$

which satisfies (1.7), (1.9), and (1.10) for $m=1$ and $n=3$. As a matter of fact, arguing as in the proof of Proposition 2.1, we have

$$
\begin{aligned}
24 \mathcal{F}\left(u^{2}\right) & =24 \mathcal{F}\left(\left(1+|x|^{2}\right)^{-4}\right) \\
& =8 \pi^{3 / 2}\left(\frac{|\xi|}{2}\right)^{5 / 2} K_{5 / 2}(|\xi|) \\
& =\left(|\xi|^{2}+3|\xi|+3\right) \mathcal{F}\left(\left(1+|x|^{2}\right)^{-2}\right) \\
& =\mathcal{F}(-\Delta u+3 \sqrt{-\Delta} u+3 u) .
\end{aligned}
$$

REMARK 3. Note, however, that in (2.12) the condition $[m]>n / 2$ is not satisfied since $m=1$ and $n=3$. This seems to confirm the technical nature of this assumption and the fact that it is not really necessary for the validity of (1.7) and (1.9). Moreover, the examples in this section seem to confirm the optimality of the estimates (1.7), (1.9), and also the more general conjecture $u(x)=O\left(|x|^{-m-n}\right)$ for the solutions of (1.1).

\section{$\S 3$. Commutator identities and estimates}

In this section we prove some commutator identities for Fourier multipliers which will be used in the proof of our result. We first state a simple but crucial assertion on the compensation of the singularities at $\xi=0$ for homogeneous symbols.

Lemma 3.1. Let $p(\xi)$ be of the form (1.4) satisfying (1.5), and let $m$ be defined by (1.6). Then the following estimates hold:

$$
\sup _{\xi \in \mathbb{R}^{n}} \frac{\left|D_{\xi}^{\sigma}\left(\xi^{\tilde{\gamma}} D_{\xi}^{\gamma} p(\xi)\right)\right|}{|p(\xi)|}<+\infty, \quad \gamma, \tilde{\gamma}, \sigma \in \mathbb{N}^{n},|\gamma|=|\tilde{\gamma}|,|\sigma| \leq[m] .
$$

Proof. Since $|\tilde{\gamma}|=|\gamma|$, then $D_{\xi}^{\sigma}\left(\xi^{\tilde{\gamma}} D_{\xi}^{\gamma} p(\xi)\right)$ is a sum of terms with homogeneity of order $m_{j}-|\sigma|$. Since $m_{j} \leq M$, we have $m_{j}-|\sigma| \leq M$. Moreover, 
in the nonpolynomial case, in view of the assumptions $|\sigma| \leq[m], m_{j} \geq m$, we have $m_{j}-|\sigma| \geq m-[m] \geq 0$. Therefore, for some $C>0$, we have

$$
\left|D_{\xi}^{\sigma}\left(\xi^{\tilde{\gamma}} D_{\xi}^{\gamma} p(\xi)\right)\right| \leq C\langle\xi\rangle^{M}, \quad \xi \in \mathbb{R}^{n} .
$$

Hence (3.1) follows from (1.5).

REMARK 4. In Lemma 3.1, and often in the rest of this article, we consider higher-order derivatives of the nonpolynomial terms $D_{\xi}^{\gamma} p_{m_{j}}(\xi)$. These derivatives should be performed in the distribution sense, possibly producing $\delta$ distribution or its derivatives at the origin. However, in all the expressions, multiplication by monomials $\xi^{\alpha}$ appears as well, so that on the whole we will always obtain a distribution $h \in \mathcal{S}^{\prime}\left(\mathbb{R}^{n}\right)$ homogeneous of order larger than $-n$. Then $\delta$ contributions are cancelled. Strictly speaking, the distribution $h \in \mathcal{S}^{\prime}\left(\mathbb{R}^{n}\right)$ can be identified in this case with the function $h_{\mathbb{R}^{n} \backslash 0} \in C^{\infty}\left(\mathbb{R}^{n} \backslash 0\right) \cap L_{\text {loc }}^{1}\left(\mathbb{R}^{n}\right)$. (Let us refer, e.g., to [18, Chapter 1, Section 3.11] for a detailed explanation.) Summing up, in Lemma 3.1 and in the rest of this article we may limit ourselves to argue in classical terms, that is, on the pointwise definition of derivatives.

Proposition 3.2. Let $p(D)$ be a Fourier multiplier defined by a homogeneous symbol $p(\xi)$ of order $m \geq 0$, and let $\alpha, \beta \in \mathbb{N}^{n}$ with $|\beta| \leq|\alpha|$. Then, for every $u \in \mathcal{S}\left(\mathbb{R}^{n}\right)$, we have the identity

$$
\begin{aligned}
& x^{\beta} p(D) D^{\alpha} u=p(D)\left(x^{\beta} D^{\alpha} u\right) \\
& +\sum_{0 \neq \gamma \leq \beta} \sum_{\tilde{\alpha}, \tilde{\beta}} C_{\alpha \beta \gamma \tilde{\alpha} \tilde{\beta} \tilde{\gamma}} D^{\tilde{\gamma}} \circ\left(D_{\xi}^{\gamma} p\right)(D)\left(x^{\tilde{\beta}} D_{x}^{\tilde{\alpha}} u\right), \\
& |\tilde{\beta}| \leq|\tilde{\alpha}|<|\alpha|
\end{aligned}
$$

where for every $\gamma$ in the sums above, $\tilde{\gamma}$ denotes a multi-index depending on $\alpha, \beta, \tilde{\alpha}, \tilde{\beta}, \gamma$ and satisfying the condition $|\tilde{\gamma}|=|\gamma|$ and $C_{\alpha \beta \gamma \tilde{\alpha} \tilde{\beta} \tilde{\gamma}}$ are suitable constants.

Proof. We can write

$$
\begin{aligned}
x^{\beta} p(D) D^{\alpha} u= & \sum_{\gamma \leq \beta}\left(\begin{array}{l}
\beta \\
\gamma
\end{array}\right) \int_{\mathbb{R}^{n}} \int_{\mathbb{R}^{n}} e^{i(x-y) \xi}(x-y)^{\gamma} p(\xi) y^{\beta-\gamma} D_{y}^{\alpha} u(y) d y \bar{d} \xi \\
= & p(D)\left(x^{\beta} D^{\alpha} u\right) \\
& +\sum_{0 \neq \gamma \leq \beta}\left(\begin{array}{c}
\beta \\
\gamma
\end{array}\right) \int_{\mathbb{R}^{n}} \int_{\mathbb{R}^{n}} D_{\xi}^{\gamma}\left(e^{i(x-y) \xi}\right) p(\xi) y^{\beta-\gamma} D_{y}^{\alpha} u(y) d y \bar{d} \xi .
\end{aligned}
$$


Integration by parts with respect to $y$ and $\xi$ gives

$$
\begin{aligned}
\int_{\mathbb{R}^{n}} & \int_{\mathbb{R}^{n}} D_{\xi}^{\gamma}\left(e^{i(x-y) \xi}\right) p(\xi) y^{\beta-\gamma} D_{y}^{\alpha} u(y) d y \bar{d} \xi \\
= & \int_{\mathbb{R}^{n}} \int_{\mathbb{R}^{n}} D_{\xi}^{\gamma}\left(-D_{y}\right)^{\alpha}\left(e^{i(x-y) \xi} y^{\beta-\gamma}\right) p(\xi) u(y) d y \bar{d} \xi \\
= & \sum_{\delta \leq \alpha}(-1)^{|\gamma|}(-i)^{|\delta|}\left(\begin{array}{c}
\alpha \\
\delta
\end{array}\right) \frac{(\beta-\gamma) !}{(\beta-\gamma-\delta) !} \\
& \quad \times \int_{\mathbb{R}^{n}} \int_{\mathbb{R}^{n}} e^{i(x-y) \xi} \xi^{\alpha-\delta}\left(D_{\xi}^{\gamma} p\right)(\xi) y^{\beta-\gamma-\delta} u(y) d y \bar{d} \xi
\end{aligned}
$$

Now let $\tilde{\gamma}$ be a multi-index such that $\tilde{\gamma} \leq \alpha-\delta$ and $|\tilde{\gamma}|=|\gamma|$. Such a multiindex exists since $|\gamma| \leq|\beta-\delta| \leq|\alpha-\delta|$ in the sums above. Then, write

$$
e^{i(x-y) \xi} \xi^{\alpha-\delta}=\xi^{\tilde{\gamma}}\left(-D_{y}\right)^{\alpha-\delta-\tilde{\gamma}} e^{i(x-y) \xi}
$$

and integrate by parts again with respect to $y$. We obtain

$$
\begin{aligned}
\int_{\mathbb{R}^{n}} & \int_{\mathbb{R}^{n}} e^{i(x-y) \xi} \xi^{\alpha-\delta}\left(D_{\xi}^{\gamma} p\right)(\xi) y^{\beta-\gamma-\delta} u(y) d y \bar{d} \xi \\
= & \int_{\mathbb{R}^{n}} \int_{\mathbb{R}^{n}} e^{i(x-y) \xi} \xi^{\tilde{\gamma}}\left(D_{\xi}^{\gamma} p\right)(\xi) D_{y}^{\alpha-\delta-\tilde{\gamma}}\left(y^{\beta-\gamma-\delta} u(y)\right) d y \bar{d} \xi \\
= & \sum_{\theta \leq \alpha-\delta-\tilde{\gamma}}(-i)^{|\theta|}\left(\begin{array}{c}
\alpha-\delta-\tilde{\gamma} \\
\theta
\end{array}\right) \frac{(\beta-\gamma-\delta) !}{(\beta-\gamma-\delta-\theta) !} \\
& \times \int_{\mathbb{R}^{n}} \int_{\mathbb{R}^{n}} e^{i(x-y) \xi} \xi^{\tilde{\gamma}}\left(D_{\xi}^{\gamma} p\right)(\xi) y^{\beta-\gamma-\delta-\theta} D_{y}^{\alpha-\tilde{\gamma}-\delta-\theta} u(y) d y \bar{d} \xi,
\end{aligned}
$$

which gives (3.2).

Proposition 3.3. Let $q(D)$ be a Fourier multiplier defined by a homogeneous symbol $q(\xi)$ of order $m>0$. Then, for every $\rho \in \mathbb{N}^{n}$ with $|\rho|<m+n$ and for every $v \in \mathcal{S}\left(\mathbb{R}^{n}\right)$, the following identity holds:

$$
x^{\rho} q(D) v=q(D)\left(x^{\rho} v\right)+\sum_{0 \neq \sigma \leq \rho}\left(\begin{array}{l}
\rho \\
\sigma
\end{array}\right)(-1)^{|\sigma|}\left(D_{\xi}^{\sigma} q\right)(D)\left(x^{\rho-\sigma} v\right) .
$$


Proof. Note that the condition $|\sigma|<m+n$ and the homogeneity imply that $D_{\xi}^{\sigma} q(\xi) \in L_{\text {loc }}^{1}\left(\mathbb{R}^{n}\right)$. Then, integrating by parts, we have

$$
\begin{aligned}
x^{\rho} q(D) v & =\int_{\mathbb{R}^{n}}\left(D_{\xi}^{\rho} e^{i x \xi}\right) q(\xi) \hat{v}(\xi) \bar{d} \xi \\
& =\sum_{\sigma \leq \rho}\left(\begin{array}{c}
\rho \\
\sigma
\end{array}\right)(-1)^{|\sigma|} \int_{\mathbb{R}^{n}} e^{i x \xi} D_{\xi}^{\sigma} q(\xi) D_{\xi}^{\rho-\sigma} \hat{v}(\xi) \bar{d} \xi
\end{aligned}
$$

from which (3.3) follows.

For fixed $s \in \mathbb{R}$, we will denote by $H_{1}^{s}\left(\mathbb{R}^{n}\right)$ the space of all $u \in \mathcal{S}^{\prime}\left(\mathbb{R}^{n}\right)$ such that

$$
\|u\|_{H_{1}^{s}}:=\left\|\langle D\rangle^{s} u\right\|_{L^{1}}<\infty .
$$

The next result states some useful estimates for singular operators, that is, operators with symbol $q(\xi) \rightarrow \infty$ for $\xi \rightarrow 0$.

LEMmA 3.4. Let $q(\xi) \in C^{\infty}\left(\mathbb{R}^{n} \backslash 0\right)$ be a homogeneous symbol of order $\mu \in(-n / 2,0)$, and let $\varphi \in C_{o}^{\infty}\left(\mathbb{R}^{n}\right)$ such that $\varphi(\xi)=1$ for $|\xi| \leq 1$. Consider the operator

$$
\begin{aligned}
H_{\varphi, q} v(x) & :=((\varphi q)(D) v)(x) \\
& =\int_{\mathbb{R}^{n}} \int_{\mathbb{R}^{n}} e^{i(x-y) \xi} \varphi(\xi) q(\xi) v(y) d y \bar{d} \xi, \quad v \in \mathcal{S}\left(\mathbb{R}^{n}\right) .
\end{aligned}
$$

Then we have

$$
\left\|H_{\varphi, q} v\right\|_{H^{s}} \leq C_{s}\|v\|_{H_{1}^{s}}
$$

Proof. Observe that we can write $\varphi(\xi) q(\xi)=q(\xi)-(1-\varphi(\xi)) q(\xi)$. Since $q(\xi)$ is a homogeneous distribution of order $\mu$, then its inverse Fourier transform is a homogeneous distribution of order $-n-\mu$. On the other hand, it is immediate to check that the inverse Fourier transform of $(1-\varphi(\xi)) q(\xi)$ is rapidly decreasing. Then we have

$$
\left|\mathcal{F}_{\xi \rightarrow x}^{-1}(\varphi(\xi) q(\xi))(x)\right| \leq C\langle x\rangle^{-n-\mu} .
$$

Since $\mu>-n / 2$, the estimate above implies that $\mathcal{F}_{\xi \rightarrow x}^{-1}(\varphi(\xi) q(\xi))(x) \in$ $L^{2}\left(\mathbb{R}^{n}\right)$. Hence, writing

$$
H_{\varphi, q} v(x)=\left(\mathcal{F}_{\xi \rightarrow x}^{-1}(\varphi(\xi) q(\xi)) * v\right)(x),
$$

the estimate (3.4) follows as a consequence of the Young inequality. 
Now we address the case of commutation with fractional powers.

LEMMA 3.5. Let $q(\xi)$ be a smooth positively homogeneous symbol of order $\mu$, let $r \in(0,1)$, and let $\varphi \in C_{o}^{\infty}\left(\mathbb{R}^{n}\right)$ such that $\varphi(\xi)=1$ for $|\xi| \leq 1$. If $\mu-r>$ $-n / 2$, then for every $v \in \mathcal{S}\left(\mathbb{R}^{n}\right)$ we have

$$
\left\|\left[\langle x\rangle^{r}, H_{\varphi, q}\right] v\right\|_{s} \leq C_{s}\|v\|_{H_{1}^{s}} .
$$

If, moreover, $\mu-r>0$, then

$$
\left\|\left[\langle x\rangle^{r}, H_{\varphi, q}\right] v\right\|_{s} \leq C_{s}\|v\|_{s} .
$$

Proof. Writing explicitly the commutator, we have

$$
\left[\langle x\rangle^{r}, H_{\varphi, q}\right] v=\int_{\mathbb{R}^{n}} \int_{\mathbb{R}^{n}} e^{i(x-y) \xi}\left(\langle x\rangle^{r}-\langle y\rangle^{r}\right) \varphi(\xi) q(\xi) v(y) d y \bar{d} \xi .
$$

By the homogeneity properties of $q(\xi)$, arguing as in the proof of Lemma 3.4, we have that the kernel $K(x, y)$ of the operator above satisfies the estimates

$$
|K(x, y)| \leq C\langle x-y\rangle^{-n-\mu+r}
$$

and the same estimates hold for all the derivatives. In particular, if $\mu-r>$ $-n / 2$, then by the Young inequality, the operator $\left[\langle x\rangle^{r}, H_{\varphi, q}\right]$ maps continuously $L^{1}\left(\mathbb{R}^{n}\right)$ into $L^{2}\left(\mathbb{R}^{n}\right)$, whereas if $\mu-r>0$, it is bounded on $L^{2}\left(\mathbb{R}^{n}\right)$. Similarly, one can treat the derivatives and obtain Sobolev continuity and the estimates (3.5) and (3.6). The lemma is then proved.

\section{$\S 4$. Proof of the main result}

In this section we prove Theorem 1.1. We can assume without loss of generality that $F(u)=u^{k}$ for some integer $k \geq 2$ and that $p(\xi)$ is of the form (1.4) with $h=1, m=m_{1}=M$; that is, $p(\xi)=p_{0}+p_{m}(\xi)$ with $p_{m}(\xi)$ a nonpolynomial positively homogeneous function of order $m$ with $[m]>n / 2$. The extension to the general case is obvious. We first give a preliminary result.

LEMMA 4.1. Under the assumptions of Theorem 1.1, we have $u \in$ $H^{s+1,1}\left(\mathbb{R}^{n}\right)$.

Proof. We first prove that $u \in H^{s+1}\left(\mathbb{R}^{n}\right)$, that is, that $D_{j} u \in H^{s}\left(\mathbb{R}^{n}\right)$ for every $j \in\{1, \ldots, n\}$. Differentiating (1.8), we obtain

$$
P\left(D_{j} u\right)=D_{j} f+D_{j} u^{k}
$$


The assumption (1.5) and the condition $M \geq 1$ imply that $P$ is invertible with symbol $1 / p(\xi)$, and the operator $P^{-1} \circ D_{j}$ is bounded on $H^{s}\left(\mathbb{R}^{n}\right)$. Then we have

$$
D_{j} u=P^{-1}\left(D_{j} f\right)+P^{-1}\left(D_{j} u^{k}\right),
$$

and since $u^{k} \in H^{s}\left(\mathbb{R}^{n}\right)$ by Schauder's estimates, we obtain

$$
\left\|D_{j} u\right\|_{s} \leq C_{s}\left(\|f\|_{s}+\|u\|_{s}^{k}\right)<\infty
$$

Starting from the assumption $\langle x\rangle^{\varepsilon_{o}} u \in H^{s}\left(\mathbb{R}^{n}\right)$, we now prove by a bootstrap argument that $u \in H^{s, 1}\left(\mathbb{R}^{n}\right)$, that is, $\langle x\rangle u \in H^{s}\left(\mathbb{R}^{n}\right)$. First, let $\varepsilon_{1}<$ $\min \left\{\varepsilon_{o}, 1-\varepsilon_{o}\right\}$, so that $\varepsilon_{o}+\varepsilon_{1}<1$. Multiplying both sides of (1.8) by $\langle x\rangle^{\varepsilon_{o}+\varepsilon_{1}}$ and introducing commutators, we have

$$
P\left(\langle x\rangle^{\varepsilon_{o}+\varepsilon_{1}} u\right)=\left[P,\langle x\rangle^{\varepsilon_{o}+\varepsilon_{1}}\right] u+\langle x\rangle^{\varepsilon_{o}+\varepsilon_{1}} f+\langle x\rangle^{\varepsilon_{o}+\varepsilon_{1}} u^{k}
$$

and then

$$
\langle x\rangle^{\varepsilon_{o}+\varepsilon_{1}} u=P^{-1}\left[P,\langle x\rangle^{\varepsilon_{o}+\varepsilon_{1}}\right] u+P^{-1}\left(\langle x\rangle^{\varepsilon_{o}+\varepsilon_{1}} f\right)+P^{-1}\left(\langle x\rangle^{\varepsilon_{o}+\varepsilon_{1}} u^{k}\right) .
$$

Now we write explicitly the commutator

$$
\left[P,\langle x\rangle^{\varepsilon_{o}+\varepsilon_{1}}\right] u=\iint e^{i(x-y) \xi}\left(\langle y\rangle^{\varepsilon_{o}+\varepsilon_{1}}-\langle x\rangle^{\varepsilon_{o}+\varepsilon_{1}}\right) p_{m}(\xi) u(y) d y d \xi .
$$

Let $\varphi \in C_{o}^{\infty}\left(\mathbb{R}^{n}\right)$ such that $\varphi(\xi)=1$ for $|\xi| \leq 1$. Then we can decompose the commutator as

$$
\left[P,\langle x\rangle^{\varepsilon_{o}+\varepsilon_{1}}\right] u=Q_{1} u(x)+Q_{2} u(x),
$$

where

$$
Q_{1} u(x)=\iint e^{i(x-y) \xi}\left(\langle y\rangle^{\varepsilon_{o}+\varepsilon_{1}}-\langle x\rangle^{\varepsilon_{o}+\varepsilon_{1}}\right) \varphi(\xi) p_{m}(\xi) u(y) d y d \xi
$$

and

$$
Q_{2} u(x)=\iint e^{i(x-y) \xi}\left(\langle y\rangle^{\varepsilon_{o}+\varepsilon_{1}}-\langle x\rangle^{\varepsilon_{o}+\varepsilon_{1}}\right)(1-\varphi(\xi)) p_{m}(\xi) u(y) d y d \xi .
$$

By Lemma 3.5 with $q(\xi)=p_{m}(\xi), \mu=m$, and $r=\varepsilon_{o}+\varepsilon_{1}$, since $0<$ $\varepsilon_{o}+\varepsilon_{1}<1$ and the condition $[m]>n / 2$ implies that $m \geq 1$, we have $m-$ $\varepsilon_{o}-\varepsilon_{1}>0$. Then $Q_{1}$ is bounded on $H^{s}\left(\mathbb{R}^{n}\right)$, and then the same is true for $P^{-1} \circ Q_{1}$. On the other hand, $Q_{2}$ is an operator with smooth amplitude of 
order $m$. Then by the classical theory (see [13], [21]), we have that $P^{-1} \circ Q_{2}$ is bounded on $H^{s}\left(\mathbb{R}^{n}\right)$. In conclusion, we have

$$
\left\|P^{-1}\left[P,\langle x\rangle^{\varepsilon_{o}+\varepsilon_{1}}\right] u\right\|_{s} \leq C_{s}\|u\|_{s}<\infty .
$$

Moreover, by Schauder's lemma we have, since $\varepsilon_{1}<\varepsilon_{o}$,

$$
\left\|P^{-1}\left(\langle x\rangle^{\varepsilon_{o}+\varepsilon_{1}} u^{k}\right)\right\|_{s} \leq C_{s}\left\|\langle x\rangle^{\varepsilon_{o}+\varepsilon_{1}} u^{k}\right\|_{s} \leq C_{s}^{\prime}\left\|\langle x\rangle^{\varepsilon_{o}} u\right\|_{s}^{2} \cdot\|u\|_{s}^{k-2}<\infty .
$$

Hence

$$
\left\|\langle x\rangle^{\varepsilon_{o}+\varepsilon_{1}} u\right\|_{s} \leq C_{s}\left(\left\|\langle x\rangle^{\varepsilon_{o}+\varepsilon_{1}} f\right\|_{s}+\|u\|_{s}+\left\|\langle x\rangle^{\varepsilon_{o}} u\right\|_{s}^{2} \cdot\|u\|_{s}^{k-2}\right)<\infty .
$$

Then $\langle x\rangle^{\varepsilon_{o}+\varepsilon_{1}} u \in H^{s}\left(\mathbb{R}^{n}\right)$. Possibly iterating this argument a finite number of times, we obtain $\langle x\rangle^{\varepsilon} u \in H^{s}\left(\mathbb{R}^{n}\right)$ for every $\varepsilon \in(0,1)$. To obtain that $u \in H^{s, 1}\left(\mathbb{R}^{n}\right)$, we need a further step. Of course, it is sufficient to show that $x_{h} u \in H^{s}\left(\mathbb{R}^{n}\right)$ for any $h=1, \ldots, n$. Arguing as in (4.1), we have

$$
\left\|x_{h} u\right\|_{s} \leq C_{s}\left(\left\|P^{-1}\left[P, x_{h}\right] u\right\|_{s}+\left\|P^{-1}\left(x_{h} f\right)\right\|_{s}+\left\|P^{-1}\left(x_{h} u^{k}\right)\right\|_{s}\right) .
$$

Now, $P^{-1}\left[P, x_{h}\right]$ is the Fourier multiplier with symbol $\frac{\left(D_{\xi_{h}} p\right)(\xi)}{p(\xi)}$ which is bounded on $H^{s}\left(\mathbb{R}^{n}\right)$. Moreover,

$$
\left\|P^{-1}\left(x_{h} u^{k}\right)\right\|_{s} \leq C_{s}\left\|x_{h} u^{k}\right\|_{s} \leq C_{s}^{\prime}\left\|\langle x\rangle u^{k}\right\|_{s} \leq C_{s}^{\prime \prime}\left\|\langle x\rangle^{1 / 2} u\right\|_{s}^{2} \cdot\|u\|_{s}^{k-2}<\infty
$$

by the previous step. Then we obtain that $x_{h} u \in H^{s}\left(\mathbb{R}^{n}\right), h=1, \ldots, n$, that is, that $u \in H^{s, 1}\left(\mathbb{R}^{n}\right)$. Finally, we prove that $x_{h} D_{j} u \in H^{s}\left(\mathbb{R}^{n}\right)$ for every $h, j \in$ $\{1, \ldots, n\}$, that is, that $u \in H^{s+1,1}\left(\mathbb{R}^{n}\right)$. Starting from (1.8) and arguing as before, we get

$$
x_{h} D_{j} u=P^{-1}\left(x_{h} D_{j} f\right)+P^{-1}\left(x_{h} D_{j} u^{k}\right)+P^{-1}\left[P, x_{h}\right] D_{j} u .
$$

Clearly we have $\left\|P^{-1}\left(x_{h} D_{j} f\right)\right\|_{s}<\infty$. Moreover,

$$
\begin{aligned}
\left\|P^{-1}\left(x_{h} D_{j} u^{k}\right)\right\|_{s} & \leq C_{s}\left(\left\|P^{-1} \circ D_{j}\left(x_{h} u^{k}\right)\right\|_{s}+\left\|P^{-1}\left[x_{h}, D_{j}\right] u^{k}\right\|_{s}\right) \\
& \leq C_{s}^{\prime}\left(\left\|\langle x\rangle u^{k}\right\|_{s}+\left\|u^{k}\right\|_{s}\right) \leq C_{s}^{\prime \prime}\|\langle x\rangle u\|_{s} \cdot\|u\|_{s}^{k-1}<\infty .
\end{aligned}
$$

Concerning the commutator, we can readily repeat the argument used before and obtain that

$$
\left\|P^{-1}\left[P, x_{h}\right] D_{j} u\right\|_{s} \leq C_{s}\left\|D_{j} u\right\|_{s}<\infty
$$

The lemma is then proved. 
Proof of Theorem 1.1. We divide the proof into two steps.

Step 1. Let us set

$$
\begin{aligned}
k_{\mathrm{cr}} & =\max \{j \in \mathbb{N}: j<m+n / 2\} \\
& = \begin{cases}{[m+n / 2]} & \text { if } m+n / 2 \notin \mathbb{N}, \\
m+n / 2-1 & \text { if } m+n / 2 \in \mathbb{N} .\end{cases}
\end{aligned}
$$

We first prove that $u \in C^{\infty}\left(\mathbb{R}^{n}\right)$ and $D^{\alpha} u \in H^{s,|\alpha|+k_{\text {cr }}}\left(\mathbb{R}^{n}\right)$ for every $\alpha \in \mathbb{N}^{n}$. This is equivalent to showing that, for every fixed $\alpha, \beta, \rho \in \mathbb{N}^{n}$, with $|\beta| \leq|\alpha|$ and $|\rho| \leq k_{\mathrm{cr}}$, we have $x^{\rho+\beta} D^{\alpha} u \in H^{s}\left(\mathbb{R}^{n}\right)$. This will be proved by induction on $|\rho+\alpha|$. For $|\rho+\alpha|=1$, the assertion is given by Lemma 4.1. Assume now that $x^{\rho+\beta} D^{\alpha} u \in H^{s}\left(\mathbb{R}^{n}\right)$ for $|\rho| \leq k_{\mathrm{cr}},|\beta| \leq|\alpha|$, and $|\rho+\alpha| \leq N$ for some positive integer $N$, and let us prove the same for $|\rho+\alpha|=N+1$. We first apply $x^{\beta} D^{\alpha}$ to both sides of (1.8) and introduce commutators. We obtain

$$
P\left(x^{\beta} D^{\alpha} u\right)=x^{\beta} D^{\alpha} f+x^{\beta} D^{\alpha} u^{k}-\left[x^{\beta} D^{\alpha}, P\right] u .
$$

By Proposition 3.2, we get

$$
\begin{aligned}
P\left(x^{\beta} D^{\alpha} u\right)= & x^{\beta} D^{\alpha} f+x^{\beta} D^{\alpha} u^{k} \\
& -\sum_{0 \neq \gamma \leq \beta} \sum_{\substack{\tilde{\alpha}, \tilde{\beta} \\
|\tilde{\beta}| \leq|\tilde{\alpha}|<|\alpha|}} C_{\alpha \beta \gamma \tilde{\alpha} \tilde{\beta} \tilde{\gamma}} D^{\tilde{\gamma}} \circ\left(D_{\xi}^{\gamma} p_{m}\right)(D)\left(x^{\tilde{\beta}} D^{\tilde{\alpha}} u\right),
\end{aligned}
$$

where $|\tilde{\gamma}|=|\gamma|$. We now multiply both sides of (4.3) by $x^{\rho}$ and write

$$
P\left(x^{\rho+\beta} D^{\alpha} u\right)=x^{\rho} P\left(x^{\beta} D^{\alpha} u\right)+\left[P, x^{\rho}\right]\left(x^{\beta} D^{\alpha} u\right) .
$$

We have, by Proposition 3.3,

$$
\begin{aligned}
& P\left(x^{\rho+\beta} D^{\alpha} u\right)=x^{\rho+\beta} D^{\alpha} f+x^{\rho+\beta} D^{\alpha} u^{k} \\
& -\sum_{0 \neq \sigma \leq \rho}\left(\begin{array}{l}
\rho \\
\sigma
\end{array}\right)(-1)^{|\sigma|}\left(D_{\xi}^{\sigma} p_{m}\right)(D)\left(x^{\rho-\sigma+\beta} D^{\alpha} u\right) \\
& -\sum_{0 \neq \gamma \leq \beta} \sum_{\tilde{\alpha}, \tilde{\beta}} C_{\alpha \beta \gamma \tilde{\alpha} \tilde{\beta} \tilde{\gamma}} x^{\rho} D^{\tilde{\gamma}} \circ\left(D_{\xi}^{\gamma} p_{m}\right)(D)\left(x^{\tilde{\beta}} D^{\tilde{\alpha}} u\right) . \\
& |\tilde{\beta}| \leq|\tilde{\alpha}|<|\alpha|
\end{aligned}
$$


Applying again Proposition 3.3 with $q(\xi)=\xi^{\tilde{\gamma}} D_{\xi}^{\gamma} p_{m}(\xi)$ and resetting the sums, we obtain for new constants $C_{\alpha \beta \gamma \tilde{\alpha} \tilde{\beta} \tilde{\gamma} \rho \sigma}$

$$
\begin{aligned}
& P\left(x^{\rho} x^{\beta} D^{\alpha} u\right) \\
& =x^{\rho+\beta} D^{\alpha} f+x^{\rho+\beta} D^{\alpha} u^{k} \\
& \quad+\sum_{\gamma \leq \beta, \sigma \leq \rho,|\sigma| \leq[m]} \sum_{\substack{|\tilde{\beta}| \leq|\tilde{\alpha}| \leq|\alpha| \\
|\alpha|-|\tilde{\alpha}|+|\sigma|>0}} C_{\alpha \beta \gamma \tilde{\alpha} \tilde{\beta} \tilde{\gamma} \rho \sigma} p_{m}^{\gamma, \tilde{\gamma}, \sigma}(D)\left(x^{\rho-\sigma+\tilde{\beta}} D^{\tilde{\alpha}} u\right) \\
& \quad+\sum_{\gamma \leq \beta, \sigma \leq \rho,|\sigma|>m} \sum_{\substack{|\tilde{\beta}| \leq|\tilde{\alpha}| \leq|\alpha| \\
|\alpha|-|\tilde{\alpha}|+|\sigma|>0}} C_{\alpha \beta \gamma \tilde{\alpha} \tilde{\beta} \tilde{\gamma} \rho \sigma} p_{m}^{\gamma, \tilde{\gamma}, \sigma}(D)\left(x^{\rho-\sigma+\tilde{\beta}} D^{\tilde{\alpha}} u\right),
\end{aligned}
$$

where $p_{m}^{\gamma, \tilde{\gamma}, \sigma}(D)$ is the Fourier multiplier with symbol $p_{m}^{\gamma, \tilde{\gamma}, \sigma}(\xi)=$ $D_{\xi}^{\sigma}\left(\xi^{\tilde{\gamma}} D_{\xi}^{\gamma} p_{m}(\xi)\right)$. Note that if $|\sigma| \leq[m]$, then $D_{\xi}^{\sigma}\left(\xi^{\tilde{\gamma}} D_{\xi}^{\gamma} p_{m}(\xi)\right)$ is well defined and locally bounded on $\mathbb{R}^{n}$ (see Lemma 3.1). If $|\sigma|>m$, then $m-|\sigma| \geq$ $m-k_{\mathrm{cr}}>-n / 2$, and then in particular $p_{m}^{\gamma, \tilde{\gamma}, \sigma}(\xi) \in L_{\mathrm{loc}}^{1}\left(\mathbb{R}^{n}\right)$ and it defines a homogeneous distribution of order $m-|\sigma|$. Now let $\varphi \in C_{o}^{\infty}\left(\mathbb{R}^{n}\right)$ with $\varphi(\xi)=$ 1 for $|\xi| \leq 1$. For $|\sigma|>m$, we can write $p_{m}^{\gamma, \tilde{\gamma}, \sigma}(\xi)=p_{m, 1}^{\gamma, \tilde{\gamma}, \sigma}(\xi)+p_{m, 2}^{\gamma, \tilde{\gamma}, \sigma}(\xi)$, where $p_{m, 1}^{\gamma, \tilde{\gamma}, \sigma}(\xi)=(1-\varphi(\xi)) p_{m}^{\gamma, \tilde{\gamma}, \sigma}(\xi)$ and $p_{m, 2}^{\gamma, \tilde{\gamma}, \sigma}(\xi)=\varphi(\xi) p_{m}^{\gamma, \tilde{\gamma}, \sigma}(\xi)$. Then we can invert $P$ and take Sobolev norms. We get

$$
\begin{aligned}
& \left\|x^{\rho+\beta} D^{\alpha} u\right\|_{s} \leq\left\|P^{-1}\left(x^{\rho+\beta} D^{\alpha} f\right)\right\|_{s}+\left\|P^{-1}\left(x^{\rho+\beta} D^{\alpha} u^{k}\right)\right\|_{s} \\
& +\sum_{\gamma \leq \beta, \sigma \leq \rho,|\sigma| \leq[m]} \sum_{|\tilde{\beta}| \leq|\tilde{\alpha}| \leq|\alpha|}\left|C_{\alpha \beta \gamma \tilde{\alpha} \tilde{\beta} \tilde{\gamma} \rho \sigma}\right| \\
& |\alpha|-|\tilde{\alpha}|+|\sigma|>0 \\
& \cdot\left\|P^{-1} \circ p_{m}^{\gamma, \tilde{\gamma}, \sigma}(D)\left(x^{\rho-\sigma+\tilde{\beta}} D^{\tilde{\alpha}} u\right)\right\|_{s} \\
& +\sum_{\gamma \leq \beta, \sigma \leq \rho,|\sigma|>m} \sum_{\substack{|\tilde{\beta}| \leq|\tilde{\alpha}| \leq|\alpha| \\
|\alpha|-|\tilde{\alpha}|+|\sigma|>0}}\left|C_{\alpha \beta \gamma \tilde{\alpha} \tilde{\beta} \tilde{\gamma} \rho \sigma}\right| \\
& \cdot\left\|P^{-1} \circ p_{m, 1}^{\gamma, \tilde{\gamma}, \sigma}(D)\left(x^{\rho-\sigma+\tilde{\beta}} D^{\tilde{\alpha}} u\right)\right\|_{s} \\
& +\sum_{\gamma \leq \beta, \sigma \leq \rho,|\sigma|>m} \sum_{\substack{|\tilde{\beta}| \leq|\tilde{\alpha}| \leq|\alpha| \\
|\alpha|-|\tilde{\alpha}|+|\sigma|>0}}\left|C_{\alpha \beta \gamma \tilde{\alpha} \tilde{\beta} \tilde{\gamma} \rho \sigma}\right| \\
& \cdot\left\|P^{-1} \circ p_{m, 2}^{\gamma, \tilde{\gamma}, \sigma}(D)\left(x^{\rho-\sigma+\tilde{\beta}} D^{\tilde{\alpha}} u\right)\right\|_{s},
\end{aligned}
$$


where $p_{m, j}^{\gamma, \tilde{\gamma}, \sigma}(D), j=1,2$, denote the operators associated to the symbols $p_{m, j}^{\gamma, \tilde{\gamma}, \sigma}(\xi), j=1,2$. We want to estimate the five terms in the right-hand side of (4.6). The first is finite by assumption. Concerning the nonlinear term, if $\rho=\beta=0$, by the boundedness of $P^{-1} \circ D_{j}, j=1, \ldots, n$, using the Leibniz formula and Schauder's estimates, we get

$$
\begin{aligned}
\left\|P^{-1} D^{\alpha} u^{k}\right\|_{s} & \leq C_{s}\left\|D^{\alpha-e_{j}} u^{k}\right\|_{s} \\
& \leq C_{s \alpha}\|u\|_{s+|\alpha|-1}^{k}<\infty
\end{aligned}
$$

by the inductive assumption. If $\rho+\beta \neq 0$, we can write

$$
\begin{aligned}
x^{\rho+\beta} D^{\alpha} u^{k}= & k x^{\rho+\beta} u^{k-1} D^{\alpha} u \\
& +x^{\rho+\beta} \sum_{\substack{\alpha_{1}+\cdots+\alpha_{k}=\alpha \\
\left|\alpha_{j}\right|<|\alpha| \forall j}} \frac{\alpha !}{\alpha_{1} ! \cdots \alpha_{k} !} D^{\alpha_{1}} u \times \cdots \times D^{\alpha_{k}} u .
\end{aligned}
$$

Moreover, since $|\beta| \leq|\alpha|$, we can write $\beta=\beta_{1}+\cdots+\beta_{k}$ for some $\beta_{j}$ satisfying $\left|\beta_{j}\right| \leq\left|\alpha_{j}\right|, j=1, \ldots, k$. Then we have, for some $\ell \in\{1, \ldots, n\}$ :

$$
\begin{aligned}
\left\|x^{\rho+\beta} D^{\alpha} u^{k}\right\|_{s} \leq & C_{s, \alpha}\left(\left\|x^{\rho+\beta-e_{\ell}} D^{\alpha} u\right\|_{s} \cdot\left\|x_{\ell} u\right\|_{s} \cdot\|u\|_{s}^{k-2}\right. \\
& \left.+\sum_{\substack{\alpha_{1}+\cdots+\alpha_{k}=\alpha \\
\left|\alpha_{j}\right|<|\alpha| \forall j}}\left\|x^{\rho+\beta_{1}} D^{\alpha_{1}} u\right\|_{s} \cdot \prod_{j=2}^{k}\left\|x^{\beta_{j}} D^{\alpha_{j}} u\right\|_{s}\right) \\
& <\infty
\end{aligned}
$$

by the inductive assumption. The third and fourth terms in the righthand side of (4.6) can be easily estimated inductively observing that, by Lemma 3.1, the operators $P^{-1} \circ p_{m}^{\gamma, \tilde{\gamma}, \sigma}(D)$ with $|\sigma| \leq[m]$ and $P^{-1} \circ p_{m, 1}^{\gamma, \tilde{\gamma}, \sigma}(D)$ are both bounded on $H^{s}\left(\mathbb{R}^{n}\right)$ and that $|\tilde{\beta}| \leq|\tilde{\alpha}|$ and $|\rho-\sigma+\tilde{\alpha}|<|\rho+\alpha|$ since $|\alpha|-|\tilde{\alpha}|+|\sigma|>0$. Concerning the last term, the estimate is more delicate since we have to deal with singular operators. Nevertheless, we can apply Lemma 3.4 with $q(\xi)=p_{m}^{\gamma, \tilde{\gamma}, \sigma}(\xi)$ and $\mu=m-|\sigma| \geq m-k_{\mathrm{cr}}>-n / 2$. We obtain

$$
\left\|P^{-1} \circ p_{m, 2}^{\gamma, \tilde{\gamma}, \sigma}(D)\left(x^{\rho-\sigma+\tilde{\beta}} D^{\tilde{\alpha}} u\right)\right\|_{s} \leq C_{s}\left\|x^{\rho-\sigma+\tilde{\beta}} D^{\tilde{\alpha}} u\right\|_{H_{1}^{s}}
$$


Moreover,

$$
\left\|x^{\rho-\sigma+\tilde{\beta}} D^{\tilde{\alpha}} u\right\|_{H_{1}^{s}} \leq C_{s}\left\|\langle x\rangle^{|\rho|-1+|\tilde{\beta}|} D^{\tilde{\alpha}} u\right\|_{s} .
$$

As a matter of fact, we have, for some $k \in\{1, \ldots, n\}$ :

$$
\begin{aligned}
\left\|x^{\rho-\sigma+\tilde{\beta}} D^{\tilde{\alpha}} u\right\|_{L^{1}} & \leq C\left\|\langle x\rangle^{-|\sigma|+1}\langle x\rangle^{|\rho|-1+|\tilde{\beta}|} D^{\tilde{\alpha}} u\right\|_{L^{1}} \\
& \leq C\left\|\langle x\rangle^{-|\sigma|+1}\right\|_{L^{2}} \cdot\left\|\langle x\rangle^{|\rho|-1+|\tilde{\beta}|} D^{\tilde{\alpha}} u\right\|_{L^{2}} \\
& \leq C^{\prime}\left\|\langle x\rangle^{|\rho|-1+|\tilde{\beta}|} D^{\tilde{\alpha}} u\right\|_{L^{2}},
\end{aligned}
$$

by the Hölder inequality, as the condition $|\sigma|>m$ implies that $|\sigma| \geq[m]+$ $1>n / 2+1$ and this gives $\langle x\rangle^{-|\sigma|+1} \in L^{2}\left(\mathbb{R}^{n}\right)$. Similar estimates can be proved for the derivatives and give (4.7). In conclusion, we obtain

$$
\left\|P^{-1} \circ p_{m, 2}^{\gamma, \tilde{\gamma}, \sigma}(D)\left(x^{\rho-\sigma+\tilde{\beta}} D^{\tilde{\alpha}} u\right)\right\|_{s} \leq C_{s}\left\|\langle x\rangle^{|\rho|-1+|\tilde{\beta}|} D^{\tilde{\alpha}} u\right\|_{s}<\infty
$$

by the inductive assumption.

Step 2. Now let $\tau$ be the fractional part, that is, $0<\tau<1, k_{\mathrm{cr}}+\tau<$ $m+n / 2$. To conclude the proof, we need to prove that $\langle x\rangle^{\tau} x^{\rho+\beta} D^{\alpha} u \in$ $H^{s}\left(\mathbb{R}^{n}\right)$ for every $\rho, \alpha, \beta \in \mathbb{N}^{n}$ with $|\beta| \leq|\alpha|$ and $|\rho| \leq k_{\mathrm{cr}}$. Starting from the identity (4.5), multiplying both sides by $\langle x\rangle^{\tau}$ and introducing commutators, we obtain

$$
\begin{aligned}
& P\left(\langle x\rangle^{\tau} x^{\rho+\beta} D^{\alpha} u\right) \\
& =\left[P,\langle x\rangle^{\tau}\right]\left(x^{\rho+\beta} D^{\alpha} u\right)+\langle x\rangle^{\tau} P\left(x^{\rho+\beta} D^{\alpha} u\right) \\
& =\left[P,\langle x\rangle^{\tau}\right]\left(x^{\rho+\beta} D^{\alpha} u\right)+\langle x\rangle^{\tau} x^{\rho+\beta} D^{\alpha} f+\langle x\rangle^{\tau} x^{\rho+\beta} D^{\alpha} u^{k} \\
& \quad+\sum_{\gamma \leq \beta, \sigma \leq \rho,|\sigma| \leq[m]} \sum_{\substack{|\tilde{\beta}| \leq|\tilde{\alpha}| \leq|\alpha| \\
|\alpha|-|\tilde{\alpha}|+|\sigma|>0}} C_{\alpha \beta \gamma \tilde{\alpha} \tilde{\beta} \tilde{\gamma} \rho \sigma} p_{m}^{\gamma, \tilde{\gamma}, \sigma}(D)\left(\langle x\rangle^{\tau} x^{\rho-\sigma+\tilde{\beta}} D^{\tilde{\alpha}} u\right) \\
& \quad \sum_{\gamma \leq \beta, \sigma \leq \rho,|\sigma| \leq[m]} \sum_{\substack{|\tilde{\beta}| \leq|\tilde{\alpha}| \leq|\alpha| \\
|\alpha|-|\tilde{\alpha}|+|\sigma|>0}} C_{\alpha \beta \gamma \tilde{\alpha} \tilde{\beta} \tilde{\gamma} \rho \sigma}\left[\langle x\rangle^{\tau}, p_{m}^{\gamma, \tilde{\gamma}, \sigma}(D)\right]\left(x^{\rho-\sigma+\tilde{\beta}} D^{\tilde{\alpha}} u\right) \\
& \quad \sum_{\gamma \leq \beta, \sigma \leq \rho,|\sigma|>[m]} C_{\substack{|\tilde{\beta}| \leq|\tilde{\alpha}| \leq|\alpha| \\
|\alpha|-|\tilde{\alpha}|+|\sigma|>0}}
\end{aligned}
$$




$$
\begin{aligned}
& +\sum_{\gamma \leq \beta, \sigma \leq \rho,|\sigma|>[m]} \sum_{\substack{|\tilde{\beta}| \leq|\tilde{\alpha}| \leq|\alpha| \\
|\alpha|-|\tilde{\alpha}|+|\sigma|>0}} C_{\alpha \beta \gamma \tilde{\alpha} \tilde{\beta} \tilde{\gamma} \rho \sigma}\left[\langle x\rangle^{\tau}, p_{m, 1}^{\gamma, \tilde{\gamma}, \sigma}(D)\right]\left(x^{\rho-\sigma+\tilde{\beta}} D^{\tilde{\alpha}} u\right) \\
& +\sum_{\gamma \leq \beta, \sigma \leq \rho,|\sigma|>[m]} \sum_{\substack{|\tilde{\beta}| \leq|\tilde{\alpha}| \leq|\alpha| \\
|\alpha|-|\tilde{\alpha}|+|\sigma|>0}} C_{\alpha \beta \gamma \tilde{\alpha} \tilde{\beta} \tilde{\gamma} \rho \sigma}\langle x\rangle^{\tau} p_{m, 2}^{\gamma, \tilde{\gamma}, \sigma}(D)\left(x^{\rho-\sigma+\tilde{\beta}} D^{\tilde{\alpha}} u\right) .
\end{aligned}
$$

At this point we can apply $P^{-1}$ to both sides of (4.9) and take Sobolev norms. We already know that $P^{-1}$ and $P^{-1} \circ p_{m}^{\gamma, \tilde{\gamma}, \sigma}(D)$ for $|\sigma| \leq[m]$ and $P^{-1}\left[P,\langle x\rangle^{\tau}\right]$ are bounded on $H^{s}\left(\mathbb{R}^{n}\right)$. Moreover, we recall that $p_{m, 1}^{\gamma, \tilde{\gamma}, \sigma}(D)$ is a Fourier multiplier with smooth symbol of negative order; then it is bounded on $H^{s}\left(\mathbb{R}^{n}\right)$. For the same reason, since $\tau<1$, we have that $\left[\langle x\rangle^{\tau}, p_{m, 1}^{\gamma, \tilde{\gamma}, \sigma}(D)\right]$ is a pseudodifferential operator with smooth and bounded symbol; then it is also bounded on $H^{s}\left(\mathbb{R}^{n}\right)$. We obtain

$$
\begin{aligned}
& \left\|\langle x\rangle^{\tau} x^{\rho+\beta} D^{\alpha} u\right\|_{s} \\
& \leq C_{s}\left(\left\|x^{\rho+\beta} D^{\alpha} u\right\|_{s}+\left\|\langle x\rangle^{\tau} x^{\rho+\beta} D^{\alpha} f\right\|_{s}+\left\|\langle x\rangle^{\tau} x^{\rho+\beta} D^{\alpha} u^{k}\right\|_{s}\right) \\
& +\sum_{\gamma \leq \beta, \sigma \leq \rho,|\sigma| \leq[m]} \sum_{\substack{|\tilde{\beta}| \leq|\tilde{\alpha}| \leq|\alpha| \\
|\alpha|-|\tilde{\alpha}|+|\sigma|>0}} C_{s \alpha \beta \gamma \tilde{\alpha} \tilde{\beta} \tilde{\gamma} \rho \sigma} \cdot\left\|\langle x\rangle^{\tau} x^{\rho-\sigma+\tilde{\beta}} D^{\tilde{\alpha}} u\right\|_{s} \\
& +\sum_{\gamma \leq \beta, \sigma \leq \rho,|\sigma| \leq[m]} \sum_{|\tilde{\beta}| \leq|\tilde{\alpha}| \leq|\alpha|} C_{s \alpha \beta \gamma \tilde{\alpha} \tilde{\beta} \tilde{\gamma} \rho \sigma} \\
& |\alpha|-|\tilde{\alpha}|+|\sigma|>0 \\
& \cdot\left\|\left[\langle x\rangle^{\tau}, p_{m}^{\gamma, \tilde{\gamma}, \sigma}(D)\right] x^{\rho-\sigma+\tilde{\beta}} D^{\tilde{\alpha}} u\right\|_{s} \\
& +\sum_{\substack{\gamma \leq \beta, \sigma \leq \rho \\
|\sigma|>m}} \sum_{\substack{|\tilde{\beta}| \leq|\tilde{\alpha}| \leq|\alpha| \\
|\alpha|-|\tilde{\alpha}|+|\sigma|>0}} C_{s \alpha \beta \gamma \tilde{\alpha} \tilde{\beta} \tilde{\gamma} \rho \sigma}\left\|\langle x\rangle^{\tau} x^{\rho-\sigma+\tilde{\beta}} D^{\tilde{\alpha}} u\right\|_{s} \\
& +\sum_{\substack{\gamma \leq \beta, \sigma \leq \rho \\
|\sigma|>m}} \sum_{\substack{|\tilde{\beta}| \leq|\tilde{\alpha}| \leq|\alpha| \\
|\alpha|-|\tilde{\alpha}|+|\sigma|>0}} C_{s \alpha \beta \gamma \tilde{\alpha} \tilde{\beta} \tilde{\gamma} \rho \sigma}\left\|x^{\rho-\sigma+\tilde{\beta}} D^{\tilde{\alpha}} u\right\|_{s} \\
& +\sum_{\substack{\gamma \leq \beta, \sigma \leq \rho \\
|\sigma|>m}} \sum_{\substack{|\tilde{\beta}| \leq|\tilde{\alpha}| \leq|\alpha| \\
|\alpha|-|\tilde{\alpha}|+|\sigma|>0}} C_{s \alpha \beta \gamma \tilde{\alpha} \tilde{\beta} \tilde{\gamma} \rho \sigma}\left\|\langle x\rangle^{\tau} p_{m, 2}^{\gamma, \tilde{\gamma}, \sigma}(D)\left(x^{\rho-\sigma+\tilde{\beta}} D^{\tilde{\alpha}} u\right)\right\|_{s},
\end{aligned}
$$

where $C_{s \alpha \beta \gamma \tilde{\alpha} \tilde{\beta} \tilde{\gamma} \rho \sigma}$ are positive constants. Let us now estimate the terms in the right-hand side of (4.10). The first is finite by the previous step of the 
proof, the second by assumption. Concerning the nonlinear term, we can write as before

$$
\begin{aligned}
& \langle x\rangle^{\tau} x^{\rho+\beta} D^{\alpha} u^{k} \\
& =k x^{\rho+\beta} D^{\alpha} u \cdot\langle x\rangle^{\tau} u \cdot u^{k-2} \\
& \quad+\sum_{\substack{\alpha_{1}+\cdots+\alpha_{k}=\alpha \\
\left|\alpha_{j}\right|<|\alpha|}} \frac{\alpha !}{\alpha_{1} ! \cdots \alpha_{k} !} x^{\rho+\beta_{1}} D^{\alpha_{1}} u \cdot\langle x\rangle^{\tau} x^{\beta_{2}} D^{\alpha_{2}} u \cdot \prod_{j=3}^{k} x^{\beta_{j}} D^{\alpha_{j}} u
\end{aligned}
$$

where $\left|\beta_{j}\right| \leq\left|\alpha_{j}\right|, j=1, \ldots, k$, and the last product does not appear if $k=2$. Then we have the estimate

$$
\begin{aligned}
& \left\|\langle x\rangle^{\tau} x^{\rho+\beta} D^{\alpha} u^{k}\right\|_{s} \\
& \leq C_{s}\left\|x^{\rho+\beta} D^{\alpha} u\right\|_{s} \cdot\left\|\langle x\rangle^{\tau} u\right\|_{s} \cdot\|u\|_{s}^{k-2} \\
& \quad+\sum_{\substack{\alpha_{1}+\cdots+\alpha_{k}=\alpha \\
\left|\alpha_{j}\right|<|\alpha|}} \frac{\alpha !}{\alpha_{1} ! \cdots \alpha_{k} !}\left\|x^{\rho+\beta_{1}} D^{\alpha_{1}} u\right\|_{s} \cdot\left\|\langle x\rangle^{\tau} x^{\beta_{2}} D^{\alpha_{2}} u\right\|_{s} \\
& \quad \prod_{j=3}^{k}\left\|x^{\beta_{j}} D^{\alpha_{j}} u\right\|_{s} \\
& \leq C_{s}\|u\|_{s+|\alpha|, k_{\mathrm{cr}}+|\alpha|} \cdot\left\|\langle x\rangle^{\tau} u\right\|_{s} \cdot\|u\|_{s}^{k-2}+C_{s \alpha}\|u\|_{s+|\alpha|, k_{\mathrm{cr}}+|\alpha|}^{k}<\infty
\end{aligned}
$$

since $\tau<1 \leq k_{\mathrm{cr}}$. To estimate the fourth term, we observe that, since $|\alpha|-$ $|\tilde{\alpha}|+|\sigma|>0$, then, if $\sigma \neq 0$, we have

$$
\left\|\langle x\rangle^{\tau} x^{\rho-\sigma+\tilde{\beta}} D^{\tilde{\alpha}} u\right\|_{s} \leq C_{s}\left\|\langle x\rangle^{|\rho|+|\tilde{\beta}|} D^{\tilde{\alpha}} u\right\|_{s}<\infty .
$$

If $\sigma=0$, then $|\alpha|-|\tilde{\alpha}|>0$ so that $|\tilde{\beta}|+1 \leq|\tilde{\alpha}|+1 \leq|\alpha|$. Hence

$$
\begin{aligned}
\left\|\langle x\rangle^{\tau} x^{\rho+\tilde{\beta}} D^{\tilde{\alpha}} u\right\|_{s} & \leq C_{s}\left\|\langle x\rangle^{|\rho|+|\tilde{\beta}|+1} D^{\tilde{\alpha}} u\right\|_{s} \\
& \leq C_{s}^{\prime}\|u\|_{s+|\alpha|, k_{\mathrm{cr}}+|\alpha|}<\infty
\end{aligned}
$$

by the previous step. The fifth term is more delicate to estimate. After cutting off the amplitude of the commutator, we can apply Lemma 3.5 with $r=\tau, q(\xi)=p_{m}^{\gamma, \tilde{\gamma}, \sigma}(\xi), \mu=m-|\sigma|$, and since $m-|\sigma|-\tau>-n / 2$, the 
operator $\left[\langle x\rangle^{\tau}, p_{m}^{\gamma, \tilde{\gamma}, \sigma}(D)\right]$ can be written as the sum of a bounded operator on $H^{s}\left(\mathbb{R}^{n}\right)$ and a continuous operator $H_{1}^{s}\left(\mathbb{R}^{n}\right) \rightarrow H^{s}\left(\mathbb{R}^{n}\right)$. Hence we have

$$
\begin{aligned}
\left\|\left[\langle x\rangle^{\tau}, p_{m}^{\gamma, \tilde{\gamma}, \sigma}(D)\right] x^{\rho-\sigma+\tilde{\beta}} D^{\tilde{\alpha}} u\right\|_{s} & \leq C_{s}\left(\left\|x^{\rho-\sigma+\tilde{\beta}} D^{\tilde{\alpha}} u\right\|_{s}+\left\|x^{\rho-\sigma+\tilde{\beta}} D^{\tilde{\alpha}} u\right\|_{H_{1}^{s}}\right) \\
& \leq C_{s}^{\prime}\left\|\langle x\rangle^{|\rho|+|\tilde{\beta}|} D^{\tilde{\alpha}} u\right\|_{s}<\infty
\end{aligned}
$$

by the Hölder inequality, since $|\sigma|>n / 2$. The sixth and the seventh term in the right-hand side of (4.10) are obviously finite. Concerning the last term, we can write

$$
\begin{aligned}
\langle x\rangle^{\tau} p_{m, 2}^{\gamma, \tilde{\gamma}, \sigma}(D)\left(x^{\rho-\sigma+\tilde{\beta}} D^{\tilde{\alpha}} u\right)= & p_{m, 2}^{\gamma, \tilde{\gamma}, \sigma}(D)\left(\langle x\rangle^{\tau} x^{\rho-\sigma+\tilde{\beta}} D^{\tilde{\alpha}} u\right) \\
& -\left[p_{m, 2}^{\gamma, \tilde{\gamma}, \sigma}(D),\langle x\rangle^{\tau}\right]\left(x^{\rho-\sigma+\tilde{\beta}} D^{\tilde{\alpha}} u\right)
\end{aligned}
$$

and apply Lemmas 3.4 and 3.5 with $q(\xi)=p_{m}^{\gamma, \tilde{\gamma}, \sigma}(\xi), r=\tau, \mu=m-|\sigma|$. We obtain

$$
\begin{aligned}
& \left\|\langle x\rangle^{\tau} p_{m, 2}^{\gamma, \tilde{\gamma}, \sigma}(D)\left(x^{\rho-\sigma+\tilde{\beta}} D^{\tilde{\alpha}} u\right)\right\|_{s} \\
& \leq C_{s}\left\|\langle x\rangle^{\tau} x^{\rho-\sigma+\tilde{\beta}} D^{\tilde{\alpha}} u\right\|_{H_{1}^{s}} \leq C_{s}^{\prime}\left\|\langle x\rangle^{\tau-|\sigma|}\langle x\rangle^{|\rho|+|\tilde{\beta}|} D^{\tilde{\alpha}} u\right\|_{H_{1}^{s}} \\
& \quad \leq C_{s}^{\prime \prime}\left\|\langle x\rangle^{|\rho|+|\tilde{\beta}|} D^{\tilde{\alpha}} u\right\|_{s}<\infty,
\end{aligned}
$$

arguing as in the proof of (4.8). The theorem is then proved.

Acknowledgments. The authors wish to thank the referees and the editors for their valuable comments and suggestions which helped to improve the quality of this paper.

\section{REFERENCES}

[1] S. Agmon, Lectures on Exponential Decay of Second-Order Elliptic Equations: Bounds on Eigenfunctions of $N$-body Schrödinger Operators, Math. Notes 29, Princeton University Press, Princeton, 1982. MR 0745286.

[2] C. J. Amick and J. F. Toland, Uniqueness and related analytic properties for the Benjamin-Ono equation-A nonlinear Neumann problem in the plane, Acta Math. 167 (1991), 107-126. MR 1111746. DOI 10.1007/BF02392447.

[3] T. B. Benjamin, Internal waves of permanent form in fluids of great depth, J. Fluid Mech. 29 (1967), 559-592.

[4] H. A. Biagioni and T. Gramchev, Fractional derivative estimates in Gevrey spaces, global regularity and decay for solutions to semilinear equations in $\mathbb{R}^{n}$, J. Differential Equations 194 (2003), 140-165. MR 2001032. DOI 10.1016/S0022-0396(03)00197-9. 
[5] J. L. Bona and Y. A. Li, Analyticity of solitary-wave solutions of model equations for long waves, SIAM J. Math. Anal. 27 (1996), 725-737. MR 1382830. DOI $10.1137 / 0527039$.

[6] - Decay and analyticity of solitary waves, J. Math. Pures Appl. (9) 76 (1997), 377-430. MR 1460665. DOI 10.1016/S0021-7824(97)89957-6.

[7] J. L. Bona and L. Luo, Large-time asymptotics of the generalized Benjamin-OnoBurgers equation, Discrete Contin. Dyn. Syst. Ser. S 4 (2011), 15-50. MR 2746393. DOI 10.3934/dcdss.2011.4.15.

[8] N. Burq and F. Planchon, On well-posedness for the Benjamin-Ono equation, Math. Ann. 340 (2008), 497-542. MR 2357995. DOI 10.1007/s00208-007-0150-y.

[9] M. Cappiello, T. Gramchev, and L. Rodino, "Semilinear pseudo-differential equations and travelling waves" in Pseudo-differential Operators: Partial Differential Equations and Time-Frequency Analysis, Fields Inst. Commun. 52, Amer. Math. Soc., Providence, 2007, 213-238. MR 2385327.

[10] - Sub-exponential decay and uniform holomorphic extensions for semilinear pseudodifferential equations, Comm. Partial Differential Equations 35 (2010), 846877. MR 2753622. DOI 10.1080/03605300903509120.

[11] M. Cappiello and F. Nicola, Holomorphic extension of solutions of semilinear elliptic equations, Nonlinear Anal. 74 (2011), 2663-2681. MR 2776517. DOI 10.1016/j.na.2010.12.021.

[12] - Regularity and decay of solutions of nonlinear harmonic oscillators, Adv. Math. 229 (2012), 1266-1299. MR 2855093. DOI 10.1016/j.aim.2011.10.018.

[13] H. O. Cordes, The Technique of Pseudodifferential Operators, London Math. Soc. Lecture Note Ser. 202, Cambridge University Press, Cambridge, 1995. MR 1314815. DOI $10.1017 /$ CBO9780511569425.

[14] A. de Bouard and J.-C. Saut, Symmetries and decay of the generalized KadomtsevPetviashvili solitary waves, SIAM J. Math. Anal. 28 (1997), 1064-1085. MR 1466669. DOI 10.1137/S0036141096297662.

[15] A. de Laire, Minimal energy for the traveling waves of the Landau-Lifshitz equation, SIAM J. Math. Anal. 46 (2014), 96-132. MR 3148081. DOI 10.1137/130909081.

[16] A. Erdélyi, W. Magnus, F. Oberhettinger, and F. G. Tricomi, Higher Transcendental Functions, I, II, McGraw-Hill, New York, 1953. MR 0058756.

[17] G. Fonseca and G. Ponce, The IVP for the Benjamin-Ono equation in weighted Sobolev spaces, J. Funct. Anal. 260 (2011), 436-459. MR 2737408. DOI 10.1016/j.jfa.2010.09.010.

[18] I. M. Gelfand and G. E. Shilov, Generalized Functions, I: Properties and Operations, Academic Press, New York, 1964. MR 0166596.

[19] - Generalized Functions, II: Spaces of Fundamental and Generalized Functions, Academic Press, New York, 1968. MR 0230128.

[20] P. Gravejat, Asymptotics of the solitary waves for the generalized KadomtsevPetviashvili equations, Discrete Contin. Dyn. Syst. 21 (2008), 835-882. MR 2399440. DOI 10.3934/dcds.2008.21.835.

[21] J. Hounie, On the $L^{2}$ continuity of pseudodifferential operators, Comm. Partial Differential Equations 11 (1986), 765-778. MR 0837930. DOI 10.1080/03605308608820444.

[22] I. D. Iliev, E. K. Khristov, and K. P. Kirchev, Spectral Methods in Soliton Equations, Pitman Monogr. Surveys Pure Appl. Math. 73, Longman Scientific \& Technical, New York, 1994. MR 1400888. 
[23] F. Linares, D. Pilod, and G. Ponce, Well-posedness for a higher-order BenjaminOno equation, J. Differential Equations 250 (2011), 450-475. MR 2737850. DOI 10.1016/j.jde.2010.08.022.

[24] R. B. Lockhart and R. C. McOwen, On elliptic systems in $\mathbb{R}^{n}$, Acta Math. 150 (1983), 125-135; Correction, Acta Math. 153 (1984), 303-304. MR 0697610. DOI $10.1007 / B F 02392969$.

[25] M. Maris, On the existence, regularity and decay of solitary waves to a generalized Benjamin-Ono equation, Nonlinear Anal. 51 (2002), 1073-1085. MR 1926086. DOI 10.1016/S0362-546X(01)00880-X.

[26] R. C. McOwen, On elliptic operators in $\mathbb{R}^{n}$, Comm. Partial Differential Equations 5 (1980), 913-933. MR 0584101. DOI 10.1080/03605308008820158.

[27] L. Molinet, J. C. Saut, and N. Tzvetkov, Ill-posedness issues for the BenjaminOno and related equations, SIAM J. Math. Anal. 33 (2001), 982-988. MR 1885293. DOI 10.1137/S0036141001385307.

[28] H. Ono, Algebraic solitary waves in stratified fluids, J. Phys. Soc. Japan 39 (1975), 1082-1091. MR 0398275.

[29] V. S. Rabinovich, "Exponential estimates for eigenfunctions of Schrödinger operators with rapidly increasing and discontinuous potentials" in Complex Analysis and Dynamical Systems, Contemp. Math. 364, Amer. Math. Soc., Providence, 2004, 225236. MR 2099029. DOI 10.1090/conm/364/06687.

[30] M. Ruzhansky and M. Sugimoto, Smoothing properties of evolution equations via canonical transforms and comparison principle, Proc. Lond. Math. Soc. (3) 105 (2012), 393-423. MR 2959931. DOI 10.1112/plms/pds006.

[31] L. Schwartz, Théorie des distributions, Hermann, Paris, 1966. MR 0209834.

[32] T. Tao, Global well-posedness of the Benjamin-Ono equation in $H^{1}(\mathbb{R})$, J. Hyperbolic Differ. Equ. 1 (2004), 27-49. MR 2052470. DOI 10.1142/S0219891604000032.

[33] G. N. Watson, A treatise on the theory of Bessel functions, reprint of the 2nd ed., Cambridge University Press, Cambridge, 1958. MR 1349110.

\author{
Marco Cappiello \\ Dipartimento di Matematica \\ Università di Torino \\ 10123 Torino \\ Italy \\ marco.cappiello@unito.it \\ Todor Gramchev \\ Dipartimento di Matematica e Informatica \\ Università di Cagliari \\ 09124 Cagliari \\ Italy \\ todor@unica.it
}


Luigi Rodino

Dipartimento di Matematica

Università di Torino

10123 Torino

Italy

luigi.rodino@unito.it 